\title{
Dissemination of CBT for Insomnia
}

\author{
Rachel Manber ${ }^{1} \cdot$ Norah Simpson ${ }^{1}$
}

Published online: 9 July 2016

(C) Springer International Publishing AG 2016

\begin{abstract}
Dissemination of cognitive behavioral therapy for insomnia (CBTI) is essential because this effective non-pharmacological intervention for insomnia is currently underutilized. In this chapter, we review past and recent dissemination efforts aimed at (1) increasing the ranks of qualified treatment providers and (2) increasing availability of direct-to-consumer CBTI treatment products. Specifically, we describe a model for training licensed mental health clinicians to competency in delivering CBTI that is currently underway in the VA Healthcare system and results from the evaluation of this dissemination effort. We also discuss existing direct-to-consumer treatment programs that use a variety of formats and modalities, and the emerging use of technologies to deliver and enhance CBTI. While significant progress has been made in disseminating CBTI, we highlight the need for future dissemination work, including the importance of developing costeffective models that blend therapist-delivered treatment with direct-to-consumer modalities, and the need to create treatment guidelines, particularly as they apply to low therapist involvement and blended models of delivery.
\end{abstract}

This article is part of the Topical Collection on Behavioral Therapy

Rachel Manber

Rmanber@stanford.edu

Norah Simpson

nsimpson@stanford.edu

1 Department of Psychiatry \& Behavioral Sciences, Stanford University, 401 Quarry Road, Stanford, CA 94305, USA
Keywords Insomnia $\cdot$ Dissemination $\cdot$ CBTI $\cdot$ Competency training $\cdot$ Self-help $\cdot$ Direct to consumer

\section{Introduction}

Cognitive behavioral therapy for insomnia (CBTI) is a short and effective therapy that is recognized as a first-line treatment for insomnia by professional organizations [1,2]. CBTI is also highly acceptable to patients: after reading a description of CBTI and pharmacologic treatments for insomnia, patients rate CBTI more favorably [3-5]. However, despite recognition of its effectiveness and acceptability to patients, CBTI is underutilized [6]. This is largely due to two factors: underidentification of individuals that may benefit from treatment and limited access to care. One large epidemiological survey found that only one third of individuals with insomnia consulted a physician about their problem and, for more than half of those who did, insomnia was not the primary reason for the consult [7]. Further, when physicians are consulted, they rarely offer referrals to CBTI $[6,8]$. A second factor that compounds the problem of under-utilization is access to care: there are not enough therapists trained to deliver CBTI to meet the clinical need.

In order to increase access to this effective intervention, dissemination efforts have targeted both training of additional providers and dissemination of CBTI directly to consumers. This manuscript describes both aspects of dissemination. We begin with a description of a nationwide program to increase the number of available providers, which has been undertaken by the United States Department of Veterans Affairs (VA). We then describe consumer-directed products offering self-help CBTI. When available, we discuss evidence on the success of these dissemination efforts in terms of efficacy, effectiveness, and utilization. We then discuss lessons learned from 
these efforts as well as directions and challenges for future dissemination of CBTI.

\section{Dissemination to Providers}

Training to competency in any psychotherapy, including cognitive behavioral therapy for insomnia, consists of educational and role play experiences ("didactic training") as well as continued hands-on training that provides the skills necessary to administer CBTI effectively and with fidelity ("competency training"). In pre-licensure training, the didactic portion is typically offered in the form of courses and readings and the competency training is achieved during clinical practica and internships. In traditional post-licensure training, didactic learning opportunities are conducted through workshops and, more recently, through online learning.

Post-licensure competency-based training in a specialty area such as CBTI is rarely available. However, evidence suggests that didactic training alone is insufficient for sustainable skillful administration of psychotherapy [9] and that adding a competency training component enhances training outcomes $[10,11]$.

Recognizing the strong evidence base supporting the efficacy of CBTI, and the need for both didactic and competency training components, the Veterans Health Administration has rolled out a nationwide initiative to provide competencybased training in CBTI to licensed mental health clinicians $[12,13]$. The training has been offered to Veterans Administration (VA) mental health clinicians who routinely provide psychotherapy across multiple disciplines, including psychiatry, psychology, social work, and nursing. The VA CBTI dissemination initiative (roll out) provides a model for dissemination of CBTI to licensed mental health clinicians in different clinical settings. The treatment protocol being disseminated promotes flexible administration of CBTI that is anchored in case formulation and includes guidelines for adapting the standard components of CBTI to patients with specific comorbidities, including chronic pain, depression, and posttraumatic stress disorder (PTSD) [13].

This VA training includes a didactic training phase followed by a competency training period, during which feedback on sample work is provided by expert CBTI consultants and additional experiential learning opportunities are provided. The didactic training phase incorporates lectures, demonstrations, and experiential learning opportunities and utilizes a treatment manual developed specifically for this roll-out initiative [13]. The competency training period lasts 4 months and consists of weekly 90-min telephone consultation sessions with a CBTI expert (Treatment Consultant or TC), which are conducted in small groups of four training participants (TPs). This phase helps TPs consolidate what they have learned during the didactic portion of the training and to further improve their
CBTI delivery skills. An essential component of the competency training period is opportunity to receive corrective feedback from the TC based on a close reviewing of the TP's work samples.

Successful completion of the VA CBTI training requires the demonstration of competency in administering CBTI. Two competency rating scales and competency criteria were developed by the VA CBTI roll-out team for this purpose [14]. One scale determines competency of the initial insomnia assessment and the other rates competency in the delivery of the treatment components to patients in a collaborative therapeutic manner. The therapy competency rating scale includes items covering content specific skills (e.g., administration of the specific components of CBTI), case conceptualization, and non-specific therapeutic elements (e.g., collaboration).

To date, 598 mental health providers have successfully completed the training. Retention of TPs during the training was high. For example, the vast majority (92\%) of the first 102 TPs who began training have completed the training and met competency criteria [15]. Importantly, three quarters of those trained continued to provide CBTI to veterans 6 months after successfully completing the competency training period [16]. Program evaluation reveals that veterans treated during the competency training period have experienced clinically meaningful reductions in insomnia severity, with large preto post-treatment Cohen's $d$ effect sizes [17]. Additional benefits experienced by patients who were treated during the skillenhancing competency training phase included reduction in depression severity and suicidal ideation as well as improved quality of life $[15,18]$. These results provide evidence that CBTI can be effectively disseminated to mental health professionals in clinical settings, with very favorable patient outcomes.

The program evaluation of the VA CBTI dissemination initiative underscores the importance of including competency-based training into the dissemination. Evaluation of 6-month follow-up data revealed increases in therapists' self-efficacy between the end of the didactic training and the end of the consultation period, with a large effect size for an increase in therapists' skill-specific self-efficacy and a smaller, yet significant, increase in general psychotherapy self- efficacy [16]. This is important because Bandura's work on self-efficacy predicts that increasing therapists' selfefficacy in CBTI will contribute to its continued use and will therefore support its sustainable implementation in the VA.

The VA system has also made deliberate efforts to promote the long-term sustainable use of CBTI by encouraging treatment consultants (TCs) to provide small-scale local training that is consistent with the spirit and standards of the centralized initiative. Further, the initiative has expanded to include the identification, selection, and further training of outstanding initiative-trained therapists (treatment providers or TPs) to become treatment consultants. Training of TPs to become TCs 
includes more advanced didactic focused on sleep disorders, information about the consultation process, experience in review and ratings of assessment and therapy sessions, and discussion on how to provide constructive feedback to TPs. Mirroring the competency training period of the TPs, the skills of the newly trained TCs are further enhanced through weekly consultation with an expert TC while they work with their first cohort of TPs. To additionally support sustainability and fidelity, the initiative provides virtual office hours, which are led by a TC and provide opportunities for mental health clinicians who were trained in the initiative to ask questions about continued implementation of CBTI, including questions about challenging clinical presentations and about sleep more generally. The initiative has also created other resources, including presentations about CBTI and the VA CBTI protocol and a centralized intranet access to treatment-related resources.

Historically, the VA has led the country in establishing credentials for practice of psychology and in promoting the role of psychologists as health-care practitioners in the USA. It is now the largest employer of mental health clinicians nationally. To the best of our knowledge, it is also the first healthcare system that has adopted CBTI system-wide, as evidenced by the explicit requirement (VHA Handbook section 1160.05) that all facilities have the capacity to provide CBTI. The demonstrated success of the VA CBTI dissemination might promote similar adoption of CBTI as an essential component of health services nationwide.

\section{Dissemination Directly to Patients}

This approach, loosely considered "self-help" treatment, includes no or low provider involvement. Some of these directto-consumer options include static treatment resources such as books, booklets, videos, and educational websites. Other direct-to-consumer options offer interactive approaches, such as mobile apps and internet-based programs, where treatment is personalized based on individual presentation at baseline and where recommendations dynamically change as treatment progresses. Some of these interactive approaches include lowintensity guidance in some form, either through a "live" provider or an animated character (e.g., "sleep prof" [19]).

In addition to the differences in the treatment approaches identified above (i.e., static or interactive, with or without provider guidance), the direct-to-consumer CBTI programs also vary with respect to delivery format and structure (e.g., written materials, audio). They also vary with respect to their content (the specific cognitive and/or behavioral components offered) and with respect to pacing (i.e., how the treatment is distributed), for example, with new segments available to consumers each week $[20,21]$ or with access to the entire intervention at the beginning of treatment, as is the case with books. This heterogeneity in approaches, content, structure, and delivery format/platform has presented a challenge in assessing the overall effectiveness of the direct-to-consumer approach to CBTI dissemination.

Two meta-analyses of self-help CBTI programs conducted in the last 8 years found that direct-to-consumer CBTI treatments are effective, with the first reporting small to moderate effect sizes [22] and the second finding medium to large effects [23]. The first of these meta-analyses included 10 randomized control studies comparing direct-to-consumer (selfhelp) CBTI interventions to waitlist and to no-treatment control groups [22]. The second (2015) meta-analysis of direct-toconsumer interventions added 10 more recent studies, six of which evaluated internet-based CBTI [23]. The reasons for the comparably larger effect sizes in the more recent metaanalysis are unclear but may relate to the fact that more than half of the new trials utilized internet-based treatments, most of which were interactive and dynamic and, hence, potentially more engaging and effective.

When considering the generalizability of results from these meta-analyses to a "real-world" patient, it is important to note that most studies did not conduct intent-to-treat analysis and some had strikingly low dropout rates. In real life, patients may start but not complete treatment for a variety of reasons, including loss of interest, finding the treatment too challenging, insufficient or unsatisfactory progress, etc. This means that the actual effectiveness rates of direct-to-consumer CBTI programs in real-world settings are likely lower than reported in these meta-analyses. Indeed, a recent metaanalysis of 11 randomized controlled trials of internetdelivered CBTI ( $n=1460$ participants) concluded that this mode of delivery is effective [24•] and found that larger dropout rates were associated with smaller effect sizes.

This meta-analysis also found that interventions with longer treatment duration had larger effect sizes. Together these findings suggest that the format and duration of treatment, as well as a program's ability to retain consumers, will play a role in determining the outcomes of internet-based CBTI treatments in real-world settings.

Evidence from the earlier self-help meta-analysis by Ho and colleagues and a 2016 meta-analysis of only internetbased CBTI trials also suggest that self-help programs that provided additional support or guidance, for example, through brief telephone contact, were more effective. In the metaanalysis by Ho and colleagues, direct-to-consumer CBTI interventions that included brief points of contact with a therapist or research staff (by phone or via email) were more effective; Zachariaea and colleagues similarly found that a higher degree of added support (ranging from fully automated messaging to personal contact) were associated with larger effect sizes [24•]. It appears that even email or text-delivered support moderately improves the benefits of internet-delivered CBTI $[25,26]$, which may be particularly important for individuals with higher depressive symptom severity at baseline [27]. At a 
minimum, automated and personal contacts increase clarity and offer reminders to enhance engagement and encourage adherence to treatment recommendations. Personal contact also provides several key therapeutic elements that may contribute to the more favorable outcomes. These include accountability to another person [28], support during what is sometimes a challenging process, and opportunity to highlight gains that the patient has not recognized. While these nonspecific therapeutic elements are likely related to treatment success, the best, most cost-effective way to provide them is not yet clear.

In the last 3 years, research on direct-to-consumer CBTI programs has been mostly on internet delivery, and has examined questions beyond efficacy, including effects of these interventions on outcomes beyond sleep and effects relative to other delivery modes (e.g., group CBTI). In addition to reduction in insomnia severity, findings from this recent research suggest that internet delivery of CBTI contributes to reduction in depressive symptom severity [29] as well as to small reductions in sleep medication use [26, 29]. Additionally, a small non-inferiority study comparing guided-internet-based CBTI and group CBTI found large and comparable pre- to posttreatment, and pre-treatment to 6-month follow-up, effect sizes for insomnia severity [30]. As the internet will likely play an increasingly central role in health-care services, we expect that the availability of internet-based CBTI programs will continue to grow, as will research assessing these programs' efficacy, and effectiveness, including consumer satisfaction.

At the cutting edge of direct-to-consumer dissemination of CBTI is the mobile app platform. At the time of this publication, there are multiple apps in different stages of development and some already in use; but, to the best of our knowledge, the efficacy of CBTI delivered via mobile apps has not been established in controlled trials. Most existing apps provide a convenient vehicle for tracking sleep, education about sleep, and reminders (e.g., to record sleep diary data). While not an exhaustive review, we highlight several examples below to illustrate current uses and features of available CBTI mobile apps. Some apps are designed for use as a complement to a therapist-delivered CBTI. For example, the VHA has developed a "CBT-iCoach" app (http://www.ptsd.va. gov/public/materials/apps/cbti-coach-app.asp) that is designed to be used as a complement to therapist-delivered CBTI, with a goal of facilitating and enhancing engagement in a clinician-delivered CBTI [31]. Initial evaluation of CBTiCoach suggests that providers perceive it favorably [31]; further research is needed to ascertain the additive benefit of the app relative to therapist-delivered CBTI alone with respect to consumers' engagement, outcome, and convenience. Several online CBTI-based programs, such as Sleepio (www.sleepio. com), utilize apps to track sleep or facilitate delivery of the online CBTI program. Based in part on the growing reach of technology, we expect that interactive direct-to-consumer treatment options for CBTI, including app-based models, will continue to increase. The scope of technology in these interventions will also likely expand to include and integrate wearable and under the mattress sleep-tracking technologies into the initial sleep assessment and the development of individualized treatment recommendations.

The array of currently available direct-to-consumer treatment options provides consumers with options across and within treatment modality (book, online, or app-based approaches to CBTI treatment). Consumers' final choice will likely depend on their preference of treatment modality as well as their initial impression of the treatment package (e.g., appeal of the website or book) and the price. Ideally, this decision-making process should also include considerations about the specific manifestations of insomnia, prior treatment history, and current treatment goals.

\section{Discussion}

In this paper, we have conceptualized dissemination of CBTI in terms of increasing both the ranks of qualified treatment providers and availability of direct-to-consumer products. We described a successful model for training already licensed mental health clinician to competency in delivering CBTI and discussed attributes and features of existing direct-toconsumer treatment programs that use a variety of formats and modalities. Another way to increase access to therapistdelivered CBTI that we have not yet discussed involves the use of telehealth services to extend the reach of already trained providers to patients with no or limited access to an office visit due to logistical factors, distance from available CBTI specialists, or limitations to mobility. Initial evidence suggests that this is an effective mode of delivery [32,33]. However, at the time of this writing, telehealth delivery and validated internetdelivered CBTI and technology are not covered by most commercial health insurance companies. This is unfortunate given that the current level of access to this effective treatment still does not meet the demands for care.

We believe that further dissemination of CBTI requires work beyond continued training of providers and development of direct-to-consumer CBTI products. For example, there is a need to develop new, cost-effective, models of treatment. One example that has been proposed but not yet tested is stepped care, where patients begin with a lower intensity and cost option that usually does not involve contact with a therapist (e.g., direct-to-consumer CBTI) before moving to a higher-intensity treatment that involves a therapist [19]. Another direction to support future dissemination of CBTI is educating the public and front-line providers about $\mathrm{CBTI}$ and how to gain access to it. 
Future work in the continued dissemination of CBTI should also include the development of treatment guidelines for direct-to-consumer and for blended models of delivery, such as stepped care. At a minimum, there is a need for guidelines for assessing and screening consumers before commencing self-help CBTI. Such guidelines can help identify when direct-to-consumer CBTI programs that do not involve a clinician's guidance may be insufficient. For example, when a circadian rhythm sleep-wake disorder better explains an individual's sleep difficulties, a treatment other than CBTI is needed and the consumer should be recommended for further assessment.

Guidelines are also needed for helping consumers (or referring providers) decide when it is clinically appropriate to transition from a low- into a higher-intensity CBTI treatment (e.g., from an online treatment to working with a CBTI specialist). Related to the decision about when to transition to a higher-intensity care is the question of how to determine treatment success. While consumer satisfaction may, at face value, appear to be the best metric for success, it may not be the most appropriate, as suggested by recent evidence that outcomes that rely on patient (consumer) satisfaction may risk under-treatment of consumers relative to clinical standards [34]. Therefore, guidelines for dissemination of CBT should also include recommendations on how to best determine treatment success and when the course of treatment is complete.

\section{Conclusion}

In the last 10 years, access to CBTI treatment has increased exponentially through dissemination efforts geared to increase the number of treatment providers trained to competency and through the development of a range of direct-to-consumer treatment programs. There is still work to be done to further these dissemination efforts in order to meet clinical needs and to help consumers and front-line health-care providers navigate the expanding breadth of treatment delivery options. We are excited about the rapid progress to date in increasing awareness of, and access to, this effective treatment and believe this growth will stimulate development and testing of guidelines and novel costeffective treatment options to further enhance future dissemination of CBTI.

\section{Compliance with Ethical Standards}

Conflict of Interest Rachel Manber and Norah Simpson declare that they have no conflict of interest.

Human and Animal Rights and Informed Consent This article does not contain any studies with human or animal subjects performed by any of the authors.

\section{References}

Papers of particular interest, published recently, are highlighted as:

- Of importance

1. National Institutes of Health. National Institutes of Health State of the Science Conference statement on manifestations and management of chronic insomnia in adults, June 13-15, 2005. Sleep. 2005;28(9):1049-57.

2. Wilson SJ et al. British Association for Psychopharmacology consensus statement on evidence-based treatment of insomnia, parasomnias and circadian rhythm disorders. J Psychopharmacol. 2010;24(11):1577-601.

3. Azad $\mathrm{N}$ et al. Hospitalized patients' preference in the treatment of insomnia: pharmacological versus non-pharmacological. Can J Clin Pharmacol. 2003;10(2):89-92.

4. Morin CM et al. Patients' acceptance of psychological and pharmacological therapies for insomnia. Sleep. 1992;15(4):302-5.

5. Vincent N, Lionberg C. Treatment preference and patient satisfaction in chronic insomnia. Sleep. 2001;24(4):411-7.

6. Ancoli-Israel S, Lieberman JA. 3rd, Insomnia in primary care: overcoming diagnostic and treatment barriers. Introduction. Postgrad Med. 2004;116(6 Suppl Insomnia):4-6.

7. Estivill E. Behaviour of insomniacs and implication for their implications for their management. Sleep Med Rev. 2002;6:S3-6.

8. Morin CM et al. Epidemiology of insomnia: prevalence, self-help treatments, consultations, and determinants of help-seeking behaviors. Sleep Med. 2006;7(2):123-30.

9. Oxman AD et al. No magic bullets: a systematic review of 102 trials of interventions to improve professional practice. CMAJ. 1995;153(10):1423-31.

10. Crits-Christoph $\mathrm{P}$ et al. Training in cognitive, supportive-expressive, and drug counseling therapies for cocaine dependence. J Consult Clin Psychol. 1998;66(3):484-92.

11. Miller WR et al. A randomized trial of methods to help clinicians learn motivational interviewing. J Consult Clin Psychol. 2004;72(6):1050-62.

12. Manber R et al. Dissemination of CBTI to the non-sleep specialist: protocol development and training issues. J Clin Sleep Med. 2012;8(2):209-18.

13. Manber $\mathrm{R}$ et al. Cognitive behavioral therapy for insomnia in veterans: therapist manual. Washington, DC: U.S. Department of Veterans Affairs; 2014.

14. Manber R, the VA CBT-I Training Development Team. Competency rating scale for CBTI treatment. Washington, DC: U.S. Department of Veterans Affairs; 2010.

15. Karlin BE et al. National Dissemination of Cognitive Behavioral Therapy for Insomnia in Veterans: therapist- and patient-level outcomes. J Consult Clin Psychol. 2013;81(5):912-7.

16. Manber R et al. Lessons learned from the National Dissemination of Cognitive Behavioral Therapy for Insomnia in the Veterans Health Administration: impact of training on therapists' self-efficacy and attitudes. Sleep Med Clin. 2013;8:399-405.

17. Karlin BE et al. National evaluation of the effectiveness of cognitive behavioral therapy for insomnia among older versus younger veterans. Int J Geriatr Psychiatry. 2015;30(3):308-15.

18. Trockel $\mathrm{M}$ et al. Effects of cognitive behavioral therapy for insomnia on suicidal ideation in veterans. Sleep. 2015;38(2):259-65.

19. Espie CA. "Stepped care": a health technology solution for delivering cognitive behavioral therapy as a first line insomnia treatment. Sleep. 2009;32(12):1549-58. 
20. Ritterband LM et al. Efficacy of an Internet-based behavioral intervention for adults with insomnia. Arch Gen Psychiatry. 2009;66(7): 692-8.

21. Trockel $\mathrm{M}$ et al. An e-mail delivered CBT for sleep-health program for college students: effects on sleep quality and depression symptoms. J Clin Sleep Med. 2011;7(3):276-81.

22. van Straten A, Cuijpers P. Self-help therapy for insomnia: a metaanalysis. Sleep Med Rev. 2009;13(1):61-71.

23. Ho FY et al. Self-help cognitive-behavioral therapy for insomnia: a meta-analysis of randomized controlled trials. Sleep Med Rev. 2015;19:17-28.

24. Zachariaea $\mathrm{R}$ et al. Efficacy of internet-delivered cognitive-behavioral therapy for insomnia - a systematic review and meta-analysis of randomized controlled trials. Sleep Med Rev. 2016;30:1-10. This paper is important because it summarizes the literature on the efficacy of internet-based CBT-I programs.

25. Lancee $\mathrm{J}$ et al. Motivational support provided via email improves the effectiveness of internet-delivered self-help treatment for insomnia: a randomized trial. Behav Res Ther. 2013;51(12):797-805.

26. Kaldo V et al. Guided internet cognitive behavioral therapy for insomnia compared to a control treatment - a randomized trial. Behav Res Ther. 2015;71:90-100.
27. Lancee $\mathrm{J}$ et al. The effect of support on internet-delivered treatment for insomnia: does baseline depression severity matter? Behav Ther. 2014;45(4):507-16.

28. Mohr DC, Cuijpers P, Lehman K. Supportive accountability: a model for providing human support to enhance adherence to eHealth interventions. J Med Internet Res. 2011;13(1):e30.

29. Blom $\mathrm{K}$ et al. Internet treatment addressing either insomnia or depression, for patients with both diagnoses: a randomized trial. Sleep. 2015;38(2):267-77.

30. Blom $\mathrm{K}$ et al. Internet-vs. group-delivered cognitive behavior therapy for insomnia: a randomized controlled non-inferiority trial. Behav Res Ther. 2015;70:47-55.

31. Kuhn E et al. CBT-I Coach: a description and clinician perceptions of a mobile app for cognitive behavioral therapy. J Clin Sleep Med. 2016;12(4):597-606.

32. Arnedt JT et al. Randomized controlled trial of telephone-delivered cognitive behavioral therapy for chronic insomnia. Sleep. 2013;36(3):353-62.

33. Lichstein $\mathrm{KL}$ et al. Telehealth cognitive behavior therapy for cooccurring insomnia and depression symptoms in older adults. J Clin Psychol. 2013;69(10):1056-65.

34. Vincent N, Walsh K. Stepped care for insomnia: an evaluation of implementation in routine practice. J Clin Sleep Med. 2013;9(3): 227-34. 